Article

\title{
Phenolic and Lignan Glycosides from the Butanol Extract of Averrhoa carambola L. Root
}

\author{
Qingwei Wen ${ }^{1}$, Xing Lin ${ }^{1}$, Yeqi Liu ${ }^{2}$, Xiaohui Xu ${ }^{1}$, Tao Liang ${ }^{1}$, Ni Zheng ${ }^{1}$, Kintoko Kintoko ${ }^{1}$ \\ and Renbin Huang ${ }^{1, *}$
}

1 Pharmaceutical College, Guangxi Medical University; No. 22, Shuangyong Road, Nanning, Guangxi 530021, China; E-Mails: wqw760623@163.com (Q.W.); gxLx60@163.com (X.L.); maskinjogja@yahoo.com.sg (K.)

2 Department of Pediatrics, Louisiana State University Health Sciences Center, the Research Institute for Children Children's Hospital, 200 Henry Clay Avenue, New Orleans, LA 70118, USA; E-Mail: yeqi_liu@hotmail.com

* Author to whom correspondence should be addressed; E-Mail: huangrenbin518@163.com; Tel.: +86-771-5339-805; Fax: +86-771-5358-272.

Received: 26 September 2012; in revised form: 11 October 2012 / Accepted: 12 October 2012 / Published: 19 October 2012

\begin{abstract}
Fifteen compounds, which included six chiral lignans and nine phenolic glycosides, were separated from the butanol fraction of Averrhoa carambola L. root and identified. All of the compounds, namely 3,4,5-trimethoxyphenol-1- $O-\beta$ - $D$-glucopyranoside (1), benzyl-1-O- $\beta$-D-glucopyranoside (2), (+)-5'-methoxyisolariciresinol 3 $\alpha$ - $O-\beta$ - $D$-glucopyranoside (3), (+)-isolariciresinol $3 \alpha-O-\beta$-D-glucopyranoside (4), koaburaside (5), (+)lyoniresinol $3 \alpha-O-\beta$ - $D$-glucopyranoside (6), (-)-lyoniresinol $3 \alpha-O-\beta$ - $D$-glucopyranoside (7), (-)-5'-methoxyisolariciresinol 3 $\alpha$-O- $\beta$-D-glucopyranoside (8), (-)-isolariciresinol $3 \alpha-O-\beta$ $D$-glucopyranoside (9), 3,5-dimethoxy-4-hydroxyphenyl 1- $O$ - $\beta$-apiofuranosyl $\left(1^{\prime \prime} \rightarrow 6^{\prime}\right)-O-\beta$ $D$-glucopyranoside (10), 3,4,5-trimethoxyphenyl 1-O- $\beta$-apiofuranosyl $\left(1^{\prime \prime} \rightarrow 6\right.$ ')- $\beta$-glucopyranoside (11), methoxyhydroquinone-4- $\beta$ - $D$-glucopyranoside (12), (2S)-2-O- $\beta$ - $D$-glucopyranosyl-2-hydroxyphenylacetic acid (13), 3-hydroxy-4-methoxyphenol 1-O- $\beta$ - $D$-apiofuranosyl-(1" $\left.\rightarrow 6^{\prime}\right)-O-\beta$ - $D$-glucopyranoside (14) and 4-hydroxy-3-methoxyphenol 1-O- $\beta$ - $D$ apiofuranosyl-(1" $\left.\rightarrow 6^{\prime}\right)-O-\beta$ - $D$-glucopyranoside (15) were isolated from this plant for the first time.
\end{abstract}

Keywords: phenolic and lignan glycosides; Averrhoa carambola L.; butanol extract 


\section{Introduction}

Nowdays, herbal medicine is accepted worldwide as an alternative therapy [1,2]. Averrhoa carambola L. (Oxalidaceae) is a perennial herb widely distributed in China, Taiwan, Malaysia, India, Brazil, America, etc. Its roots, have been used as a Traditional Chinese Medicine for thousands of years in the remedy of lithangiuria, arthralgia and chronic paroxysmal headache. In our previous study, both ethanol extract and polysaccharide from the roots showed hypoglycemic and antioxidant effects $[3,4]$. Many publications have also indicated that compounds from Averrhoa carambola leaves displayed hypoglycemic, hypotriglyceridemic, anti-lipid peroxidative and anti-atherogenic properties in streptozocin-induced diabetic rats [5,6].

Previous literature reports on Averrhoa carambola L. have only reported the isolation and identification of a few compounds such as $\beta$-sitosterol, lupeol and 1,5-dihydroxy-6,7-dimethoxy-2methyl-anthraquinone 3-O- $\beta$-glucopyranoside [7-10]. Therefore, this study involved separating more compounds from the herb aiming to offer better insight into its chemical constituents. In this research, the $60 \%$ aqueous ethanol (aq. EtOH) extract from Averrhoa carambola L. roots was suspended in $\mathrm{H}_{2} \mathrm{O}$ and further extracted with cyclohexane, ethyl acetate (EtOAc) and $n$-butanol $(n-\mathrm{BuOH})$, respectively. Then, the butanol extract was successively purified by open silica gel, Sephadex LH-20, ODS column and P-HPLC to obtain 15 compounds that could be useful for investigating the hypoglycemic or antioxidant substances of the plant and standardization of the butanol extract from this herb in the next step.

\section{Results and Discussion}

The $60 \%$ aq. EtOH extract of Averrhoa carambola L. root was successively separated by cyclohexane, EtOAc and $n-\mathrm{BuOH}$. From the $\mathrm{n}-\mathrm{BuOH}$ fraction, fifteen known compounds $(\mathbf{1}-\mathbf{1 5}$, listed in the Abstract, structures shown on Figures 1 and 2) were isolated and their structures confirmed by detailed FTIR, NMR $\left({ }^{1} \mathrm{H},{ }^{13} \mathrm{C}\right)$ data comparison with those in the literature [11-22]. ${ }^{13} \mathrm{C}$-NMR data of the compounds is summarized in Tables 1 and 2. ${ }^{1} \mathrm{H}-\mathrm{NMR}$ data is given in the Experimental. These nine phenolic and six lignan glycosides still not been previously isolated and identified from this plant. Just as mentioned in the Introduction, Our preliminary investigations have shown that the ethanolic extract of Averrhoa carambola L. root could relieve the lipid peroxide reactions and has a significant hypoglycemic effect in the streptozotocin-induced diabetic mice, suggesting it may be a potential hypoglycemic agent for the treatment of diabetes and its complications [3]. Compounds 6 (418.9 mg) and $7(534.7 \mathrm{mg})$, as the main chemical ingredients of the $n$ - $\mathrm{BuOH}$ extract, may play a key role in the observed anti-diabetic effect. To explore the active substances in this plant, these two compounds' hypoglycemic activities will be investigated in the near future. The structures of the compounds are shown on Figures 1 and 2. 
Figure 1. Structures of compounds 1, 2, 5 and 10-15.

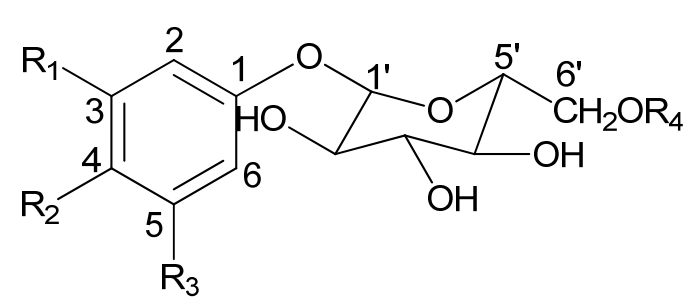

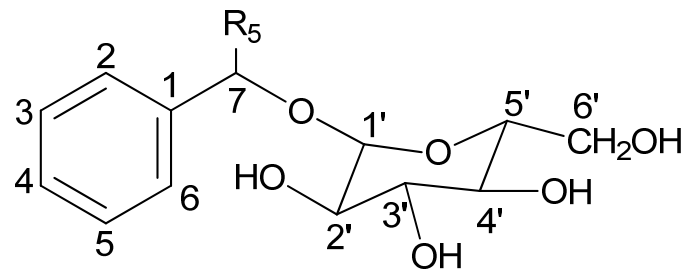

\begin{tabular}{|c|c|c|c|c|}
\hline & R1 & R2 & R3 & R4 \\
\hline 1 & -OMe & -OMe & -OMe & $-H$ \\
\hline 5 & -OMe & $-\mathrm{OH}$ & -OMe & $-\mathrm{H}$ \\
\hline 10 & -OMe & $-\mathrm{OH}$ & -OMe & \\
\hline 11 & -OMe & -OMe & -OMe & \\
\hline 12 & -OMe & $-\mathrm{OH}$ & $-\mathrm{H}$ & $-\mathrm{H}$ \\
\hline 14 & $-\mathrm{OH}$ & -OMe & $-\mathrm{H}$ & \\
\hline 15 & -OMe & $-\mathrm{OH}$ & $-\mathrm{H}$ & \\
\hline
\end{tabular}

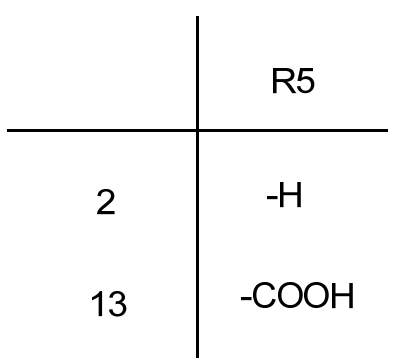

Figure 2. Structures of compounds 3, 4 and 6-9.

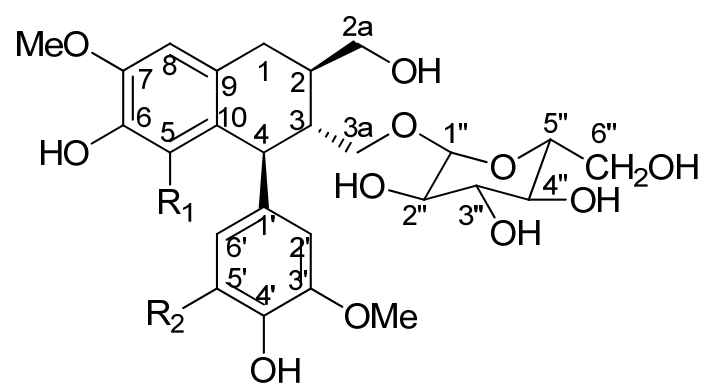<smiles>[R2]c1cc([C@H]2c3c(cc(OC)c(O)c3[R])C[C@H](CO)[C@H]2COC2COC3C(O)C(O)OC(CO)C3O2)cc(OC)c1O</smiles>

\begin{tabular}{c|cc} 
& $\mathrm{R} 1$ & $\mathrm{R} 2$ \\
\hline 3 & $\mathrm{H}$ & $-\mathrm{OMe}$ \\
4 & $\mathrm{H}$ & $\mathrm{H}$ \\
6 & $-\mathrm{OMe}$ & $-\mathrm{OMe}$
\end{tabular}

\begin{tabular}{c|cc} 
& $\mathrm{R} 1$ & $\mathrm{R} 2$ \\
\hline 7 & $-\mathrm{OMe}$ & $-\mathrm{OMe}$ \\
8 & $\mathrm{H}$ & $-\mathrm{OMe}$ \\
9 & $\mathrm{H}$ & $\mathrm{H}$
\end{tabular}


Table 1. ${ }^{13} \mathrm{C}-\mathrm{NMR}$ data of compounds $\mathbf{1 , 2}, \mathbf{5}$ and 10-15 (150 MHz, MeOD).

\begin{tabular}{|c|c|c|c|c|c|c|c|c|c|}
\hline $\mathrm{C}$ & 1 & 2 & 5 & 10 & 11 & 12 & 13 & 14 & 15 \\
\hline 1 & 156.3 & 139.1 & 156.0 & 156.0 & 134.7 & 152.9 & 138.8 & 152.7 & 155.0 \\
\hline 2 & 96.2 & 129.3 & 94.6 & 94.6 & 96.3 & 103.7 & 129.3 & 105.2 & 104.4 \\
\hline 3 & 154.8 & 129.2 & 154.8 & 154.9 & 154.9 & 149.3 & 129.3 & 149.3 & 152.1 \\
\hline 4 & 145.8 & 128.7 & 129.7 & 129.5 & 155.9 & 142.8 & 128.5 & 143.0 & 141.1 \\
\hline 5 & 154.8 & 129.2 & 154.8 & 154.9 & 154.9 & 116.0 & 129.3 & 116.3 & 120.7 \\
\hline 6 & 96.2 & 129.3 & 94.6 & 94.6 & 96.3 & 110.0 & 129.3 & 110.1 & 111.0 \\
\hline 7 & - & 71.7 & - & - & - & - & 81.4 & - & - \\
\hline R1 & 56.6 & - & 56.8 & 56.8 & 56.8 & 56.4 & - & - & 56.6 \\
\hline $\mathbf{R 2}$ & 61.2 & - & - & - & 61.3 & - & - & 56.5 & - \\
\hline R3 & 56.6 & - & 56.8 & 56.8 & 56.8 & - & - & - & - \\
\hline R5 & - & - & - & & - & - & 176.5 & - & - \\
\hline $1^{\prime}$ & 103.2 & 103.3 & 106.2 & 105.1 & 103.0 & 103.7 & 103.5 & 103.6 & 104.4 \\
\hline $2^{\prime}$ & 75.0 & 75.2 & 75.8 & 74.9 & 74.9 & 75.0 & 75.1 & 74.9 & 75.0 \\
\hline $3^{\prime}$ & 78.2 & 78.0 & 78.3 & 77.8 & 77.2 & 78.2 & 78.3 & 77.7 & 78.1 \\
\hline $4^{\prime}$ & 71.7 & 71.8 & 71.4 & 71.3 & 71.5 & 71.6 & 71.4 & 71.4 & 71.7 \\
\hline $5^{\prime}$ & 78.4 & 78.0 & 77.8 & 75.6 & 77.8 & 78.1 & 78.0 & 77.3 & 77.0 \\
\hline $6^{\prime}$ & 61.2 & 62.8 & 62.6 & 69.3 & 67.0 & 62.5 & 62.6 & 70.1 & 68.7 \\
\hline 1" & - & - & - & 105.9 & 105.9 & - & - & 103.9 & 111.0 \\
\hline $2^{\prime \prime}$ & - & - & - & 77.5 & 77.2 & - & - & 77.3 & 77.8 \\
\hline $3^{\prime \prime}$ & - & - & - & 77.8 & 77.8 & - & - & 77.9 & 80.6 \\
\hline $4^{\prime \prime}$ & - & - & - & 74.9 & 74.9 & - & - & 74.9 & 75.1 \\
\hline $5^{\prime \prime}$ & - & - & - & 66.8 & 61.3 & - & - & 66.8 & 65.7 \\
\hline
\end{tabular}

Table 2. ${ }^{13} \mathrm{C}-\mathrm{NMR}$ data of compounds 3, 4 and 6-9 (150 MHz, MeOD).

\begin{tabular}{ccccccc}
\hline $\mathbf{C}$ & $\mathbf{3}$ & $\mathbf{4}$ & $\mathbf{6}$ & $\mathbf{7}$ & $\mathbf{8}$ & $\mathbf{9}$ \\
\hline $\mathbf{1}$ & 33.7 & 33.8 & 33.8 & 33.8 & 33.6 & 33.6 \\
$\mathbf{2}$ & 39.1 & 39.6 & 40.6 & 41.3 & 41.1 & 41.1 \\
$\mathbf{2 a}$ & 64.6 & 64.9 & 66.2 & 66.2 & 65.6 & 65.5 \\
$\mathbf{3}$ & 45.8 & 45.9 & 46.7 & 46.6 & 45.3 & 45.4 \\
$\mathbf{3 a}$ & 68.9 & 69.6 & 71.5 & 72.0 & 70.7 & 70.8 \\
$\mathbf{4}$ & 47.9 & 47.9 & 42.8 & 43.2 & $\mathrm{~b}$ & $\mathrm{~b}$ \\
$\mathbf{5}$ & 116.8 & 117.4 & 148.7 & 148.7 & 117.3 & 117.4 \\
$\mathbf{6}$ & 145.2 & 146.0 & 138.9 & 138.9 & 145.3 & 146.0 \\
$\mathbf{7}$ & 146.5 & 147.4 & 147.6 & 147.6 & 147.4 & 147.3 \\
$\mathbf{8}$ & 112.1 & 112.5 & 107.9 & 107.8 & 112.4 & 112.5 \\
$\mathbf{9}$ & 128.6 & 128.9 & 130.2 & 130.2 & 129.2 & 129.2 \\
$\mathbf{1 0}$ & 133.9 & 134.0 & 126.4 & 126.2 & 133.7 & 133.8 \\
\hline $\mathbf{1}$ & 137.1 & 138.8 & 139.4 & 139.5 & 138.0 & 138.8 \\
$\mathbf{2}^{\prime}$ & 107.8 & 114.4 & 107.0 & 107.1 & 108.0 & 114.0 \\
$\mathbf{3}$ & 148.6 & 148.7 & 149.0 & 149.0 & 149.3 & 149.0 \\
$\mathbf{4}$ & 135.1 & 145.0 & 134.5 & 134.6 & 133.7 & 145.3 \\
$\mathbf{5}^{\prime}$ & 148.6 & 116.1 & 149.0 & 149.0 & 149.3 & 116.0 \\
$\mathbf{6}^{\prime}$ & 107.8 & 123.2 & 107.0 & 107.1 & 108.0 & 123.5 \\
\hline
\end{tabular}


Table 2. Cont.

\begin{tabular}{|c|c|c|c|c|c|c|}
\hline C & $3 *$ & 4 & 6 & 7 & 8 & 9 \\
\hline $1 "$ & 105.1 & 105.2 & 104.8 & 104.3 & 103.9 & 103.9 \\
\hline $2^{\prime \prime}$ & 75.1 & 75.2 & 75.2 & 75.1 & 75.1 & 75.0 \\
\hline $3^{\prime \prime}$ & 77.4 & 77.9 & 77.9 & 78.0 & 78.0 & 77.9 \\
\hline $4^{\prime \prime}$ & 71.8 & 71.7 & 71.7 & 71.6 & 71.5 & 71.5 \\
\hline $5^{\prime \prime}$ & 78.0 & 78.2 & 78.2 & 78.2 & 78.2 & 78.3 \\
\hline 6" & 63.0 & 62.5 & 62.8 & 62.7 & 62.5 & 62.5 \\
\hline $5-\mathrm{OCH}_{3}$ & - & - & 60.2 & 60.1 & - & - \\
\hline 7- $\mathrm{OCH}_{3}$ & 56.1 & 56.4 & 56.6 & 56.6 & 56.4 & 56.3 \\
\hline $3^{\prime}-\mathrm{OCH}_{3}$ & 56.7 & 56.5 & 56.9 & 56.9 & 56.9 & 56.6 \\
\hline $5^{\prime}-\mathrm{OCH}_{3}$ & 56.7 & - & 56.9 & 56.9 & 56.9 & - \\
\hline
\end{tabular}

\section{Experimental}

\subsection{General}

Melting points were measured without correction on a binocular microscopic X-5 melting point apparatus (Beijing, China). FTIR data were recorded on a PerkinElmer spectrophotometer (Spetrum One) equipped with a DGTS detector. ${ }^{1} \mathrm{H}$ and ${ }^{13} \mathrm{C}$-NMR data were obtained at $600 \mathrm{MHz}$ and $150 \mathrm{MHz}$, respectively, in $\mathrm{MeOD}, \mathrm{C}_{5} \mathrm{D}_{5} \mathrm{~N}$ and acetone- $d_{6}$, on a Bruker Av 600 instrument. Chemical shifts are expressed in $\delta(\mathrm{ppm})$ with TMS as internal standard. P-HPLC was run on a Shimadzu LC-8A equipped with a SPD-10A VP detector and an AQ-C18 column $(20 \times 250 \mathrm{~mm}, 10 \mu \mathrm{m})$. Open column chromatography (CC) was carried out using silica gel (200-300 mesh, Qingdao Marine Chemical Ltd., Qingdao, China), RP-18 (ODS-AQ-HG, YMG*GEL, $5.0 \times 60 \mathrm{~cm}, 12 \mathrm{~nm}$, S-50 $\mu \mathrm{m}$, Lot: 9955), and Sephadex LH-20 $(2.0 \times 60 \mathrm{~cm}, 20-100 \mu \mathrm{m}$, GE Healthcare, Uppsala, Sweden). TLC was performed on silica gel plates (Qingdao Marine Chemical Ltd.). Except for methanol $(\mathrm{MeOH})$ which was of chromatographic grade, the other reagents used were analytical grade and purchased from the Tanjin Damao Chemical Reagent Factory (Tanjin, China).

\subsection{Plant Materials}

The roots of Averrhoa carambola L. were collected from Linshan County, Guangxi Province, China, in June 2010 and were identified by Prof. Maoxiang Lai. The voucher specimen (No. 20100605) was deposited in the herbarium of the Guangxi Institute of Chinese Medicine \& Pharmaceutical Science (Guangxi, China).

\subsection{Extraction and Isolation}

The powder of air-dried roots of Averrhoa carambola L. (12 kg) was extracted three times with $60 \%$ aq. EtOH under reflux ( $96 \mathrm{~L}, 1 \mathrm{~h}$ each time). The ethanolic solution was concentrated under vacuum to yield a syrup-like extract which was suspended in $\mathrm{H}_{2} \mathrm{O}$ and then extracted with cyclohexane $(3 \times 20 \mathrm{~L})$, EtOAc $(3 \times 20 \mathrm{~L})$ and $n$-BuOH $(3 \times 20 \mathrm{~L})$, respectively. The $n$-BuOH extract $(153 \mathrm{~g})$ was 
subjected to silica gel open CC $(13 \times 100 \mathrm{~cm}, 200-300 \mathrm{mesh}, 1.5 \mathrm{~kg})$, eluting successively with a chloroform/MeOH gradient (100:0 to 0:100) to afford 13 fractions Fr.1-Fr.13.

Fr.7 (9.4 g) was subjected to silica gel CC $(6 \times 120 \mathrm{~cm}, 200-300 \mathrm{mesh}, 100 \mathrm{~g})$, eluting successively with a petroleum ether/EtOAc gradient (100:0, 2:1, 1:1, 0:100, each 1.0 L) and EtOAc/MeOH (15:0, 10:1, 5:1, 4:1, 3:1, 1:1, 0:100, each 1.0 L) to obtain seven subfractions Fr.71-Fr.77. Then Fr.72 (1.1 g), Fr.73 (3.2 g) was subjected to Sephadex LH-20 CC with MeOH and gave sub-fractions Fr.721-Fr.723, Fr.731-Fr.733, respectively. Fr.732 was further separated on a RP-18 CC, eluting with a MeOH/H${ }_{2} \mathrm{O}$ gradient $(10 \%, 30 \%, 50 \%, 70 \%, 100 \%$, each $150 \mathrm{~mL})$, and five fractions (Fr.7321-7325) were obtained from it. Compound $1\left(0.8 \mathrm{mg}, \mathrm{t}_{\mathrm{R}}=69.0 \mathrm{~min}\right)$, and compound $2\left(3.2 \mathrm{mg}, \mathrm{t}_{\mathrm{R}}=74.0 \mathrm{~min}\right)$ were isolated by P-HPLC $\left(\mathrm{MeOH} / \mathrm{H}_{2} \mathrm{O} 25: 75,8 \mathrm{~mL} / \mathrm{min}, 203 \mathrm{~nm}\right)$ from Fr.7321.

Fr.8 (16.6 g) was subjected to silica gel CC $(6 \times 120 \mathrm{~cm}, 200-300 \mathrm{mesh}, 160 \mathrm{~g})$, eluting successively with gradient petroleum ether/EtOAc (100:0, 1:1, 0:100) and EtOAc/MeOH (18:0, 10:1, 8:1, 6:1, 4:1, 2:1, 0:100) to obtain six sub-fractions Fr.81-Fr.86. Compounds 3 (70.3 mg) and 4 (12.8 mg) were obtained from Fr.84. Then Fr.83, Fr.84 were further purified successively by Sephadex LH-20 CC with $\mathrm{MeOH}$, and afforded fractions Fr.831-Fr.833, Fr.841-Fr.843. Fr.831, Fr.842 were purified by RP-18 $\mathrm{CC}$ and eluted with $\mathrm{MeOH} / \mathrm{H}_{2} \mathrm{O}$ gradient $(10 \%, 20 \%, 30 \%, 50 \%, 100 \%$, each $150 \mathrm{~mL})$. Ten fractions Fr.8311-Fr.8315, Fr.8421-8425 were obtained from Fr.831, Fr.842, respectively. After that, Fr.8311 was subjected to silica gel CC $(1.5 \times 120 \mathrm{~cm}, 200-300 \mathrm{mesh}, 30 \mathrm{~g})$, eluting successively with a dichloromethane/MeOH gradient (100:0, 20:1, 18:1, 15:1, 12:1, 9:1, 6:1, 3:1, 1:1, 0:100, each $450 \mathrm{~mL})$ to give eight sub-fractions Fr.83111-Fr.83118. Fr.83113 was further purified successively by P-HPLC $\left(\mathrm{MeOH} / \mathrm{H}_{2} \mathrm{O} 25: 75,8.0 \mathrm{~mL} / \mathrm{min}, 203 \mathrm{~nm}\right)$ to produce compound $5\left(4.2 \mathrm{mg}, \mathrm{t}_{\mathrm{R}}=13.0 \mathrm{~min}\right) . \mathrm{Fr} .8421$ was isolated by silica gel $\mathrm{CC}(1.5 \times 120 \mathrm{~cm}, 200-300 \mathrm{mesh}, 60 \mathrm{~g})$ with dichloromethane/MeOH gradient $(100: 0,20: 1,18: 1,16: 1,10: 1,0: 100$, each $200 \mathrm{~mL})$ and monitored by TLC to give eight fractions Fr.84211-84218. Fr.8422 was followed by RP-18 CC $(5.0 \times 60 \mathrm{~cm})$ and eluted with a $\mathrm{MeOH} / \mathrm{H}_{2} \mathrm{O}$ gradient $(10 \%, 20 \%, 30 \%, 50 \%, 100 \%$, each $150 \mathrm{~mL})$ to yield five fractions Fr.84221-Fr.84225. Then Fr.84215, Fr.8423 were purified in turns by P-HPLC $\left(\mathrm{MeOH} / \mathrm{H}_{2} \mathrm{O} 28: 72\right.$, $8.0 \mathrm{~mL} / \mathrm{min}, 203 \mathrm{~nm})$ to give compound $6\left(418.9 \mathrm{mg}, \mathrm{t}_{\mathrm{R}}=41.0 \mathrm{~min}\right)$, compound $7(534.7 \mathrm{mg}$, $\left.\mathrm{t}_{\mathrm{R}}=48.0 \mathrm{~min}\right)$, compound $\mathbf{8}\left(2.5 \mathrm{mg}, \mathrm{t}_{\mathrm{R}}=81.5 \mathrm{~min}\right)$, and compound $\mathbf{9}\left(1.0 \mathrm{mg}, \mathrm{t}_{\mathrm{R}}=95.5 \mathrm{~min}\right)$.

Fr.9 (12.7 g) was subjected to silica gel CC $(6 \times 120 \mathrm{~cm}, 200-300 \mathrm{mesh}, 130 \mathrm{~g})$ and eluted successively with a EtOAc/MeOH gradient (100:0, 15:1, 10:1, 8:1, 5:1, 4:1, 3:1, 2:1, 1:1, 0:100, each $1.0 \mathrm{~L})$, to give six sub-fractions Fr.91-Fr.96. Then Fr.92 (7.8 g) was separated by Sephadex LH-20 CC with $\mathrm{MeOH}$ and produced five fractions Fr.921-Fr.925. Fr.923 (4.8 g) was purified by RP-18 CC, eluting with a $\mathrm{MeOH} / \mathrm{H}_{2} \mathrm{O}$ gradient $(10 \%, 20 \%, 30 \%, 50 \%, 100 \%$, each $150 \mathrm{~mL})$ to obtain six fractions Fr.9231-Fr.9236. Then Fr.9232 was further purified by P-HPLC (MeOH/ $\left.\mathrm{H}_{2} \mathrm{O} 25: 75,8.0 \mathrm{~mL} / \mathrm{min}, 203 \mathrm{~nm}\right)$ to give compound $10\left(3.2 \mathrm{mg}, \mathrm{t}_{\mathrm{R}}=12.0 \mathrm{~min}\right)$, and compound $11\left(14.5 \mathrm{mg}, \mathrm{t}_{\mathrm{R}}=27.0 \mathrm{~min}\right)$. Fr.9231 was isolated by silica gel CC $(1.5 \times 120 \mathrm{~cm}, 200-300$ mesh, $3.5 \mathrm{~g})$ with a dichloromethane/MeOH gradient (100:0, 20:1, 16:1, 12:1, 10:1, 7:1, each $200 \mathrm{~mL})$ to afford 12 fractions Fr.92311-Fr.92322. Fr.92318, Fr.92319, Fr.92321 were further purified successively by P-HPLC $(8.0 \mathrm{~mL} / \mathrm{min}, 203 \mathrm{~nm})$ with $\mathrm{MeOH} / \mathrm{H}_{2} \mathrm{O}(16: 84,20: 80,13: 87)$, to produce compound $12\left(1.4 \mathrm{mg}, \mathrm{t}_{\mathrm{R}}=10.0 \mathrm{~min}\right)$, compound 13 $\left(17.2 \mathrm{mg}, \mathrm{t}_{\mathrm{R}}=17.0 \mathrm{~min}\right)$, compound $14\left(10.3 \mathrm{mg}, \mathrm{t}_{\mathrm{R}}=21.0 \mathrm{~min}\right)$, compound $15\left(7.6 \mathrm{mg}, \mathrm{t}_{\mathrm{R}}=23.0 \mathrm{~min}\right)$. 
3,4,5-Trimethoxyphenol-1-O- $\beta$-D-glucopyranoside (1). White needles, m.p. $201-203{ }^{\circ} \mathrm{C},[\alpha]_{\mathrm{D}}^{20}-28.0$ (c $0.3, \mathrm{MeOH}), \mathrm{IR}(\mathrm{KBr}) \mathrm{cm}^{-1}: 3274.0,2924.4,1602.8,1507.8,1466.4,1419.2,1384.7,1228.3$, 1197.1, 1166.7, 1127.9, 1074.9, 1055.5, 1036.7, 1019.1, 998.9, 835.0, 819.7, 781.4. ${ }^{1} \mathrm{H}-\mathrm{NMR}$ (MeOD) $\delta: ~ 3.42-3.47$ (4H, m, overlaped, H-2', 3', 4', 5'), 3.66 (1H, dd, $J=12.1,6.7$ Hz, H-6'a), 3.70 (3H, s, 4-OMe), $3.81(6 \mathrm{H}, \mathrm{s}, 3,5-\mathrm{OMe}), 3.92\left(1 \mathrm{H}, \mathrm{dd}, J=12.1,2.4 \mathrm{~Hz}, \mathrm{H}-6^{\prime} \mathrm{b}\right), 4.82$ (1H, d, $\left.J=7.3 \mathrm{~Hz}, \mathrm{H}-1^{\prime}\right), 6.49$ $(2 \mathrm{H}, \mathrm{s}, \mathrm{H}-2,6)[11]$.

Benzyl-1-O- $\beta$-D-glucopyranoside (2). White needles, m.p. $212.3-213.7^{\circ} \mathrm{C},[\alpha]_{\mathrm{D}}^{20}-41.0(c 0.2, \mathrm{MeOH})$, IR (KBr) cm ${ }^{-1}: 3273.6,2937.6,1604.1,1507.8,1466.5,1228.6,1196.9,1128.4,1074.9,1018.4,999.3$, 669.1. ${ }^{1} \mathrm{H}-\mathrm{NMR}(\mathrm{MeOD}) \delta$ : $7.42(2 \mathrm{H}, \mathrm{d}, J=7.4 \mathrm{~Hz}, \mathrm{H}-2,6), 7.26-7.34$ (3H, m, overlapped, H-3, 4, 5), $4.93(1 \mathrm{H}, \mathrm{d}, J=11.8 \mathrm{~Hz}, \mathrm{H}-7 \mathrm{a}), 4.68(1 \mathrm{H}, \mathrm{d}, J=11.8 \mathrm{~Hz}, \mathrm{H}-7 \mathrm{~b}), 4.36(1 \mathrm{H}, \mathrm{d}, J=7.7 \mathrm{~Hz}, \mathrm{H}-1$ '), $3.90(1 \mathrm{H}$, dd, $J=2.2,12.0 \mathrm{~Hz}, \mathrm{H}-6$ 'a), 3.68 (1H, dd, $J=5.6,12.0 \mathrm{~Hz}, \mathrm{H}-6$ 'b) [12].

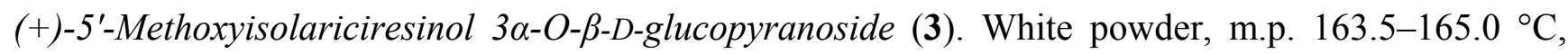
$[\alpha]_{\mathrm{D}}^{20}+11.0\left(c\right.$ 0.1, MeOH), IR (KBr) $\mathrm{cm}^{-1}:$ 3459.6, 2901.8, 1591.6, 1513.6, 1464.4, 1321.4, 1280.3, 1226.1, 1120.0, 1020.7, 949.3, 930.9. ${ }^{1} \mathrm{H}-\mathrm{NMR}$ (acetone- $\left.d_{6}\right) \delta: 7.18(1 \mathrm{H}, \mathrm{s}, \mathrm{H}-8), 7.06(1 \mathrm{H}, \mathrm{s}, \mathrm{H}-5)$, $6.54\left(2 \mathrm{H}, \mathrm{s}, \mathrm{H}-2^{\prime}, 6^{\prime}\right), 4.69$ (1H, d, $\left.J=3.7 \mathrm{~Hz}, \mathrm{H}-1^{\prime \prime}\right), 4.25(1 \mathrm{H}, \mathrm{d}, J=3.7 \mathrm{~Hz}, \mathrm{H}-3 \mathrm{a}), 4.18(1 \mathrm{H}, \mathrm{d}$, $J=4.2 \mathrm{~Hz}, \mathrm{H}-3 \mathrm{~b}), 4.09$ (1H, dd, $J=10.0,2.5 \mathrm{~Hz}, \mathrm{H}-1 \mathrm{~b}), 3.79$ (3H, s, OMe), 3.77 (6H, s, 3', 5'-OMe), 3.61-3.72 (4H, m, overlaped, H-2", 3", 4", 5"), 3.57-3.55 (1H, t), 3.41-3.37 (1H, m), 3.33 (1H, dd, $J=9.4,4.1 \mathrm{~Hz}, \mathrm{H}-6 \mathrm{a}), 3.26-3.25(3 \mathrm{H}, \mathrm{m}), 3.15$ (1H, dd, $J=10.0,2.9 \mathrm{~Hz}, \mathrm{H}-1 \mathrm{a}), 2.79$ (1H, dd, $J=16.1,4.8 \mathrm{~Hz}, \mathrm{H}-6 \mathrm{~b}), 2.08$ (1H, m, H-3) [13].

(+)-Isolariciresinol $3 \alpha-O-\beta$-D-glucopyranoside (4). White powder, m.p. $146.0-148.0{ }^{\circ} \mathrm{C},[\alpha]_{\mathrm{D}}^{20}+15.2$ (c 0.32, MeOH), IR (KBr) cm $\mathrm{cm}^{-1}$ : 3901.8, 3478.7, 1698.7, 1616.3, 1541.4, 1512.5, 1502.5, 1456.7, 1275.6, 1025.9. ${ }^{1} \mathrm{H}-\mathrm{NMR}(\mathrm{MeOD}) \delta: 6.79\left(1 \mathrm{H}, \mathrm{d}, J=2.0 \mathrm{~Hz}, \mathrm{H}-2^{\prime}\right), 6.74\left(1 \mathrm{H}, \mathrm{d}, J=8.0 \mathrm{~Hz}, \mathrm{H}-5^{\prime}\right)$, 6.65 (1H, br.s, H-8), 6.64 (1H, dd, $J=8.0,2.0$ Hz, H-6'), 4.12 (1H, d, H-3a), 4.07 (1H, t, H-3b), 3.81 $(3 \mathrm{H}, \mathrm{s}, \mathrm{OMe}), 3.80(3 \mathrm{H}, \mathrm{s}, \mathrm{OMe}), 3.77-3.70(2 \mathrm{H}, \mathrm{m}), 3.29-3.20$ (4H, m, partly overlapped), 2.86-2.78 (2H, m, H-1a, H-1b), 2.10-2.07 (1H, m, H-3), 1.85-1.88 (1H, m, H-2) [13].

Koaburaside (5). White powder, m.p. $238.0{ }^{\circ} \mathrm{C},[\alpha]_{\mathrm{D}}^{20}-21.0(c 0.25, \mathrm{MeOH}), \mathrm{IR}(\mathrm{KBr}) \mathrm{cm}^{-1}: 3704.9$, 2925.1, 1736.5, 1601.7, 1508.3, 1484.4, 1434.6, 1222.4, 1126.2, 1073.6, 996.1, 818.4, 639.0. ${ }^{1} \mathrm{H}-\mathrm{NMR}$ $(\mathrm{MeOD}) \delta: 6.13(2 \mathrm{H}, \mathrm{s}, \mathrm{H}-2,6), 3.79(6 \mathrm{H}, 2 \times \mathrm{OMe}), 4.66\left(1 \mathrm{H}, \mathrm{d}, J=7.4 \mathrm{~Hz}, \mathrm{H}-1{ }^{\prime}\right), 3.44(1 \mathrm{H}, \mathrm{dd}$, $\left.J=9.7,2.3 \mathrm{~Hz}, \mathrm{H}-2^{\prime}\right), 3.40$ (2H, dd, overlapped, H-3', 4'), 3.50-3.59 (1H, m, H-5'), 3.75 (1H, br. d, $J=17.3,8.76 \mathrm{~Hz}, \mathrm{H}-6 ' \mathrm{a}), 3.67$ (1H, dd, $J=11.8,5.2 \mathrm{~Hz}, \mathrm{H}-6 ' \mathrm{~b})$ [14].

(+)-Lyoniresinol $3 \alpha-O-\beta$-D-glucopyranoside (6). Light yellow powder, m.p. $119-120^{\circ} \mathrm{C} .[\alpha]_{\mathrm{D}}^{20}+21.0$ (c $0.4, \mathrm{MeOH}), \mathrm{IR}(\mathrm{KBr}) \mathrm{cm}^{-1}: 3365.9,2936.9,1614.7,1517.1,1500.7,1457.2,1426.5,1322.9$, 1218.7, 1111.9, 900.8, 807.0, 632.7. ${ }^{1} \mathrm{H}-\mathrm{NMR}\left(\mathrm{C}_{5} \mathrm{D}_{5} \mathrm{~N}\right) \delta: 6.73(1 \mathrm{H}, \mathrm{s}, \mathrm{H}-8), 7.05(2 \mathrm{H}, \mathrm{s}, \mathrm{H}-2$ ', H-6'), $5.14(1 \mathrm{H}, \mathrm{d}, J=6.1 \mathrm{~Hz}, \mathrm{H}-4), 4.97\left(1 \mathrm{H}\right.$, partly overlapped, H-1"), $4.52\left(1 \mathrm{H}, \mathrm{dd}, J=11.7,2.5 \mathrm{~Hz}, \mathrm{H}_{\mathrm{A}}-2 \mathrm{a}\right.$ or $\left.\mathrm{H}_{\mathrm{A}}-3 \mathrm{a}\right), 4.42\left(1 \mathrm{H}, \mathrm{dd}, J=10.0,4.3 \mathrm{~Hz}, \mathrm{H}_{\mathrm{B}}-2 \mathrm{~b}\right.$ or $\left.\mathrm{H}_{\mathrm{B}}-3 \mathrm{~b}\right), 4.36\left(1 \mathrm{H}, \mathrm{d}, J=11.8,5.3 \mathrm{~Hz}, \mathrm{H}_{\mathrm{A}}-2 \mathrm{a}\right.$ or $\mathrm{H}_{\mathrm{A}}-3 \mathrm{a}$ ), 4.26-4.22 (2H, m, H-6"), 4.14-3.94 (4H, m, overlapped, H-2",3",4",5"), 3.77 (3H, s, 7-OMe), $3.75(3 \mathrm{H}, \mathrm{s}, 5-\mathrm{OMe}), 3.71(6 \mathrm{H}, \mathrm{s}, 3,5 '-\mathrm{OMe}), 3.14(1 \mathrm{H}, \mathrm{dd}, J=14.7,12.0 \mathrm{~Hz}, \mathrm{H}-1 \mathrm{a}), 3.07(1 \mathrm{H}, \mathrm{dd}$, $J=15.2,4.4 \mathrm{~Hz}, \mathrm{H}-1 \mathrm{~b}), 2.73$ (1H, m, H-3), 2.14 (1H, m, H-2) [13,15]. 
(-)-Lyoniresinol 3 $\alpha$-O- $\beta$-D-glucopyranoside (7). Light yellow powder, m.p. $133.0-134.5{ }^{\circ} \mathrm{C},[\alpha]_{\mathrm{D}}^{20}$ -45.5 ( c 0.3, MeOH), IR (KBr) $\mathrm{cm}^{-1}: 3390.3,2936.0,1614.2,1517.3,1501.2,1461.3,1426.7,1323.9$, 1218.5, 1111.9, 901.0, 806.1, 626.2. ' ${ }^{1} \mathrm{H}-\mathrm{NMR}(\mathrm{MeOD}) \delta: 6.57$ (1H, s, H-8), 6.41 (2H, s, H-2', H-6'), $4.22(1 \mathrm{H}, \mathrm{d}, J=6.5 \mathrm{~Hz}, \mathrm{H}-4), 4.13(1 \mathrm{H}, \mathrm{d}, J=7.8 \mathrm{~Hz}, \mathrm{H}-1 "), 3.87-3.83$ (5H, m), 3.85 (3H, s, OMe), $3.75(6 \mathrm{H}, \mathrm{s}, 2 \times \mathrm{OMe}), 3.69(1 \mathrm{H}, \mathrm{dd}, J=11.9,5.5 \mathrm{~Hz}, \mathrm{H}-6$ "a). 3.63-3.57 (3H, m), 3.32-3.27 (m, mostly overlapped), 3.33 (3H, s, OMe), 3.22-3.14 (2H, m), 2.71-2.64 (2H, m, H-1a, H-1b), 2.15-2.11 (1H, m, H-3), 1.71-1.65 (1H, m, H-2) [13].

(-)-5'-Methoxyisolariciresinol 3 $\alpha$-O- $\beta$-D-glucopyranoside (8). Light yellow powder, m.p. $157.0-158.0{ }^{\circ} \mathrm{C}$, $[\alpha]_{\mathrm{D}}^{20}-40.0(c 0.5, \mathrm{MeOH}), \mathrm{IR}(\mathrm{KBr}) \mathrm{cm}^{-1}: 3420.8,2927.5,1611.7,1512.8,1456.9,1384.5,1219.2$, 1117.4. ${ }^{1} \mathrm{H}-\mathrm{NMR}(\mathrm{MeOD}) \delta$ : $6.68(1 \mathrm{H}, \mathrm{s}, \mathrm{H}-8), 6.47$ (2H, s, H-2', H-6'), 6.24 (1H, s, H-5), 4.08 (1H, d, $J=7.8 \mathrm{~Hz}, \mathrm{H}-1 "), 3.79-3.66(6 \mathrm{H}, \mathrm{m}), 3.83(3 \mathrm{H}, \mathrm{s}, \mathrm{OMe}), 3.82(6 \mathrm{H}, \mathrm{s}, 2 \times \mathrm{OMe}), 3.32-3.29(2 \mathrm{H}, \mathrm{m})$, $3.19(1 \mathrm{H}, \mathrm{dd}, J=8.9,8.0 \mathrm{~Hz}), 3.08-3.06(1 \mathrm{H}, \mathrm{m}), 2.91(1 \mathrm{H}, \mathrm{dd}, J=15.8,10.3 \mathrm{~Hz}, \mathrm{H}-1 \mathrm{a}), 2.77(1 \mathrm{H}, \mathrm{dd}$, $J=16.0,3.6 \mathrm{~Hz}, \mathrm{H}-1 \mathrm{~b}), 2.02-1.98$ (2H, m, H-2, H-3) [13].

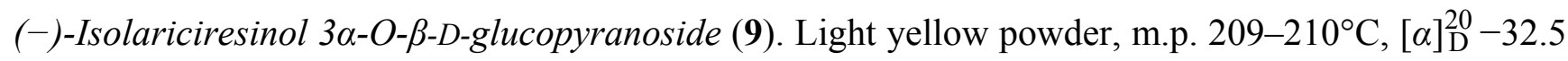
(c $0.1, \mathrm{MeOH}), \mathrm{IR}(\mathrm{KBr}) \mathrm{cm}^{-1}: 3828.9,3710.8,2924.5,2853.2,1844.5,1698.4,1594.3,1555.0$, 1541.4, 1512.6, 1464.3, 1456.8, 1384.3, 1270.9, 1125.6, 1075.2, 1033.4. ${ }^{1} \mathrm{H}-\mathrm{NMR}$ (MeOD) $\delta: 6.74$ $\left(1 \mathrm{H}, \mathrm{d}, J=8.0 \mathrm{~Hz}, \mathrm{H}-5^{\prime}\right), 6.69\left(1 \mathrm{H}, \mathrm{d}, J=1.9 \mathrm{~Hz}, \mathrm{H}-2^{\prime}\right), 6.65(1 \mathrm{H}$, br. s, H-8), $6.64(1 \mathrm{H}, \mathrm{d}, J=2.0 \mathrm{~Hz}$, H-6'), 6.19 (1H, s, H-5), 4.04 (1H, d, J=7.8 Hz, H-1"). 3.85-3.63 (m), 3.81 (3H, s, OMe), 3.80 (3H, s, OMe), 3.35-3.25 (m, partly overlapped), 3.16 (1H, m), 3.06-3.04 (1H, m), 2.89-2.86 (1H, m, H-1a), 2.75 (1H, br. dd, $J=15.4,4.0 \mathrm{~Hz}, \mathrm{H}-1 \mathrm{~b}), 1.97$ (2H, m, H-2, H-3) [13].

3,5-Dimethoxy-4-hydroxyphenyl 1-O- $\beta$-apiofuranosyl $\left(1^{\prime \prime} \rightarrow 6^{\prime}\right)-O-\beta$-D-glucopyranoside (10). Light yellow powder, m.p. $126.5-128.0{ }^{\circ} \mathrm{C},[\alpha]_{\mathrm{D}}^{20}-42.0(c 0.6, \mathrm{MeOH}), \mathrm{IR}(\mathrm{KBr}) \mathrm{cm}^{-1}: 3750.9,3725.7$, 3627.5, 3564.8, 2924.0, 1602.6, 1507.8, 1486.8, 1455.8, 1384.1, 1222.6, 1124.4, 1043.4, 818.3. ${ }^{1} \mathrm{H}-\mathrm{NMR}$ (MeOD) $\delta: 6.13(2 \mathrm{H}, \mathrm{s}, \mathrm{H}-2,6), 3.80(6 \mathrm{H}, \mathrm{s}, 3,5-\mathrm{OMe}), 4.24(1 \mathrm{H}, \mathrm{d}, J=7.4 \mathrm{~Hz}, \mathrm{H}-1$ '), 3.47-3.38 (6H, m, overlapped), $3.82(1 \mathrm{H}, \mathrm{dd}, J=11.5,5.3 \mathrm{~Hz}, \mathrm{H}-6 \mathrm{a}), 4.00(1 \mathrm{H}, \mathrm{dd}, J=11.5,2.1 \mathrm{~Hz}, \mathrm{H}-6$ 'b), 4.65 (1H, d, $J=7.7 \mathrm{~Hz}, \mathrm{H}-1 "), 3.76-3.72$ (2H, m, overlapped) [16].

3,4,5-Trimethoxyphenyl 1-O- $\beta$-apiofuranosyl $\left(1^{\prime \prime} \rightarrow 6^{\prime}\right)$ - $\beta$-glucopyranoside (11). White needles, m.p. 203.0-205.0 ${ }^{\circ} \mathrm{C},[\alpha]_{\mathrm{D}}^{20}-62.0\left(c\right.$ 0.8, MeOH), IR (KBr) cm ${ }^{-1}: 3748.6,3335.5,1698.9,1616.8,1541.3$, 1512.0, 1473.0, 1052.7. ${ }^{1} \mathrm{H}-\mathrm{NMR}(\mathrm{MeOD}) \delta: 6.47$ (2H, s, H-2, 6), 3.82 (6H, s, 3, 5-OMe), 3.71 (3H, s, 4-OMe), 4.28 (1H, d, $\left.J=7.6 \mathrm{~Hz}, \mathrm{H}-1^{\prime}\right), 4.82$ (1H, d, $\left.J=7.6 \mathrm{~Hz}, \mathrm{H}-1 "\right), 3.84$ (2H, dd, $J=11.4,5.3 \mathrm{~Hz}$, H-4") [17].

Methoxyhydroquinone-4- $\beta$-D-glucopyranoside (12). White powder, m.p. $211.0-213.0{ }^{\circ} \mathrm{C},[\alpha]_{\mathrm{D}}^{20}-34.5$ (c 0.15, MeOH), IR (KBr) $\mathrm{cm}^{-1}$ : 3748.9, 3728.4, 2923.8, 1716.0, 1576.1, 1541.3, 1513.0, 1456.7, 1383.1, 1296.7, 1244.4, 1223.7, 1197.3, 1169.4, 1082.3, 1045.7, 989.4, 942.4, 840.1, 804.6. ${ }^{1} \mathrm{H}-\mathrm{NMR}$ (MeOD) $\delta: 6.80(1 \mathrm{H}, \mathrm{d}, J=2.7 \mathrm{~Hz}, \mathrm{H}-5), 6.69(1 \mathrm{H}, \mathrm{d}, J=8.6 \mathrm{~Hz}, \mathrm{H}-2), 6.59(1 \mathrm{H}, \mathrm{dd}, J=8.6,2.7 \mathrm{~Hz}$, H-6), 3.83 (3H, s, OMe), 4.74 (1H, d, $J=7.4 \mathrm{~Hz}, \mathrm{H}-1$ '), 3.46-3.35 (4H, overlapped), 3.90 (1H, dd, $J=12.0,2.2 \mathrm{~Hz}, \mathrm{H}-6 \mathrm{a}$ ) , 3.69 (1H, dd, $J=12.0,5.8 \mathrm{~Hz}, \mathrm{H}-6$ 'b) [18,19]. 
(2S)-2-O- $\beta$-D-Glucopyranosyl-2-hydroxyphenylacetic acid (13). Amorphous powder, m.p. 117.0-119.0 ${ }^{\circ} \mathrm{C}$, $[\alpha]_{\mathrm{D}}^{20}-87.5\left(c\right.$ 0.4, MeOH), IR (KBr) $\mathrm{cm}^{-1}:$ 3748.7, 3628.3, 3176.7, 2883.1, 1676.1, 1590.1, 1555.3, 1541.6, 1512.0, 1497.0, 1456.0, 1411.5, 1308.1, 1196.4, 1156.2, 1076.0, 897.1, 766.7, 702.8. ${ }^{1} \mathrm{H}-\mathrm{NMR}$

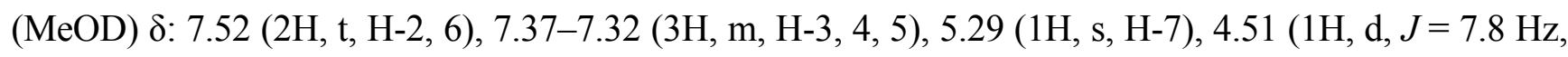
H-1'), 3.87 (1H, d, $J=11.8 \mathrm{~Hz}, \mathrm{H}-6$ 'a), 3.67 (1H, dd, $J=12.0,5.0 \mathrm{~Hz}, \mathrm{H}-6$ 'b), 3.46-3.40 (1H, m), 3.35 $(1 \mathrm{H}, \mathrm{d}, J=8.0 \mathrm{~Hz})[20,21]$.

3-Hydroxy-4-methoxyphenol 1-O- $\beta$-D-apiofuranosyl-( $\left(1^{\prime \prime} \rightarrow 6^{\prime}\right)-O-\beta$-D-glucopyranoside (14). White powder, m.p. 226.0-228.0 ${ }^{\circ} \mathrm{C},[\alpha]_{\mathrm{D}}^{20}-47.5$ (c 0.3, MeOH), IR (KBr) $\mathrm{cm}^{-1}: 3391.5,1618.4,1513.9,1384.6$, 1226.6, 1201.5, 1114.3, 1070.9, 856.2. ${ }^{1} \mathrm{H}-\mathrm{NMR}(\mathrm{MeOD}) \delta: 6.76(1 \mathrm{H}, \mathrm{d}, J=9.0 \mathrm{~Hz}, \mathrm{H}-5), 6.71(1 \mathrm{H}, \mathrm{d}$, $J=2.4 \mathrm{~Hz}, \mathrm{H}-2), 6.62(1 \mathrm{H}, \mathrm{dd}, J=8.4,3.0 \mathrm{~Hz}, \mathrm{H}-6), 3.84(3 \mathrm{H}, \mathrm{s}, 4-\mathrm{OMe}), 4.31(1 \mathrm{H}, \mathrm{d}, J=7.2 \mathrm{~Hz}$, H-1'), 3.61-3.58 (1H, m), 3.48-3.44 (1H, m), $3.79(1 \mathrm{H}, \mathrm{dd}, J=11.4,6.0 \mathrm{~Hz}, \mathrm{H}-6 \mathrm{a}), 4.10$ (1H, dd, $J=11.4,1.8 \mathrm{~Hz}, \mathrm{H}-6 \mathrm{\prime}$ ) $), 4.74$ (1H, d, $J=7.2 \mathrm{~Hz}, \mathrm{H}-1 "), 3.42-3.37$ (3H, m, overlapped), 3.21-3.14 (3H, m, overlapped) [22].

4-Hydroxy-3-methoxyphenol 1-O- $\beta$-D-apiofuranosyl-(1" $\left.\rightarrow 6^{\prime}\right)-O-\beta$-D-glucopyranoside $\quad$ (15). Light yellow powder, m.p. $124.0-125.0{ }^{\circ} \mathrm{C},[\alpha]_{\mathrm{D}}^{20}-54.0(c 0.3, \mathrm{MeOH}), \mathrm{IR}(\mathrm{KBr}) \mathrm{cm}^{-1}: 3750.8,3725.8$, 3654.8, 3627.8, 2926.8, 1675.1, 1605.6, 1541.5, 1512.5, 1456.6, 1384.7, 1301.8, 1214.3, 1164.0, 1063.5, 954.2, 834.0, 807.0. ${ }^{1} \mathrm{H}-\mathrm{NMR}(\mathrm{MeOD}) \delta: 7.00(1 \mathrm{H}, \mathrm{d}, J=8.7 \mathrm{~Hz}, \mathrm{H}-2), 6.47(1 \mathrm{H}, \mathrm{d}, J=2.8 \mathrm{~Hz}, \mathrm{H}-5)$, $6.32(1 \mathrm{H}, \mathrm{dd}, J=8.5,2.8 \mathrm{~Hz}, \mathrm{H}-6), 3.81(3 \mathrm{H}, \mathrm{s}, 3-\mathrm{OMe}), 4.66\left(1 \mathrm{H}, \mathrm{d}, J=7.5 \mathrm{~Hz}, \mathrm{H}-1^{\prime}\right), 3.43-3.42$ (2H, m, overlapped), 3.48-3.45 (1H, m, overlapped), 3.62 (1H, dd, $J=11.1,6.4 \mathrm{~Hz}, \mathrm{H}-6 \mathrm{a}), 3.98$ (1H, $\mathrm{dd}, J=11.1,1.9 \mathrm{~Hz}, \mathrm{H}-6 ' \mathrm{~b}), 4.98$ (1H, d, $J=2.3 \mathrm{~Hz}, \mathrm{H}-1 "), 3.89$ (1H, d, $J=2.3 \mathrm{~Hz}, \mathrm{H}-2 "), 3.74$ (1H, d, $J=9.6 \mathrm{~Hz}, \mathrm{H}-4$ "a), 3.93 (1H, d, $J=9.7 \mathrm{~Hz}, \mathrm{H}-4 " \mathrm{~b}), 3.58$ (2H, s) [16].

\section{Conclusions}

The ${ }^{1} \mathrm{H}$ and ${ }^{13} \mathrm{C}-\mathrm{NMR}$ data of 15 compounds isolated from the roots of Averrhoa carambola $\mathrm{L}$. for the first time were identical with the literature values.

\section{Supplementary Materials}

FTIR, ${ }^{1} \mathrm{H}$ and ${ }^{13} \mathrm{C}-\mathrm{NMR}$ spectrum of compounds $\mathbf{1 - 1 5}$ are available as Supplementary Materials which can be accessed at: http://www.mdpi.com/1420-3049/17/10/12330/s1.

\section{Acknowledgments}

This work was supported by National Natural Science Foundation of China (No. 81160533); Guangxi Natural Science Foundation (No. 2012GXNSFAA053106, No. 0832013Z).

\section{References}

1. Seidell, J.C. Obesity, Insulin Resistance and Diabetes-a Worldwide Epidemic. Br. J. Nutr. 2000, $83, \mathrm{~S} 5-\mathrm{S} 8$. 
2. Diez, J.J.; Iglesias, P. The role of the novel adipocyte derived hormone adiponectin in human disease. Eur. J. Endocrinol. 2003, 148, 293-300.

3. Huang, G.H.; Huang, R.B. Effects of Alcoholic Extracts of Averrhoa carambola L. Root on Blood Glucose Level and Lipid Peroxidation in Diabetic Mice. Lishizhen Med. Mater. Med. Res. 2009, 20, 2730-2731.

4. Huang, G.H.; Huang, C.Z.; Huang, R.B. Effects of Averrhoa Carambola L. Root Polysaccharide on Serum Insulin and Index of Thymus, Spleen in STZ-induced Diabetic Mice. Chin. Pharm. 2009, 12, 848-850.

5. Cazarolli, L.H.; Folador, P.; Moresco, H.H.; Brighente, I.M.; Pizzolatti, M.G.; Silva, F.R. Stimulatory effect of apigenin-6-C- $\beta$-L-fucopyranoside on insulin secretion and glycogen synthesis. Eur. J. Med. Chem. 2009, 44, 4668-4673.

6. Cazarolli, L.H.; Folador, P.; Moresco, H.H.; Brighente, I.M.; Pizzolatti, M.G.; Silva, F.R. Mechanism of action of the stimulatory effect of apigenin- $6-C-\left(2^{\prime \prime}-O-\alpha-L-r h a m n o p y r a n o s y l\right)-\beta-L-$ fucopyranoside on ${ }^{14} \mathrm{C}$-glucose uptake. Chem. Biol. Interact. 2009, 179, 407-412.

7. Ranganayaki, S.; Singh, R.; Singh, A.K. The chemical examination of the bark of A. carambola. Proc. Natl. Acad. Sci. India A 1980, 50, 61-63.

8. Gunasegaran, R. Flavonoids and anthocyanins of three Oxalidaceae. Fitoterapia 1992, 63, 89-90.

9. Araho, D.; Miyakoshi, M.; Chou, W.; Kambara, T.; Mizutani, K.; Ikeda, T. A new flavone C-glycoside from the leaves of Averrhoa carambola. Nat. Med. 2005, 59, 113-116.

10. Moresco, H.H.; Queiroz, G.S.; Pizzolatti, M.G.; Brighente, I. Chemical constituents and evaluation of the toxic and antioxidant activities of Averrhoa carambola leaves. Rev. Bras. Farmacogn. 2012, 22, 319-324.

11. Shimomura, H.; Sashida, Y.; Oohara, M.; Tenma, H. Phenolic Glucosides from Parabenzoin Praecox. Phytochemistry 1988, 27, 644-645.

12. Zeng, Q.; Chang R.J.; Qin, J.J.; Cheng, X.G.; Zhang, W.D.; Jin, H.Z. New Glycosides from Dracocephalum tanguticum Maxim. Arch. Pharm. Res. 2011, 34, 2015-2020.

13. Achenbach, H.; Löwel, M.; Waibel, R.; Gupta, M.; Soils, P. New Lignan Glucosides from Stemmadenia minima. Planta Med. 1992, 58, 270-272.

14. Chung, M.I.; Lai, M.H.; Yen, M.H.; Wu, R.R.; Lin, C.N. Phenolics from Hypericum geminiflorum. Phytochemistry 1997, 44, 943-947.

15. Zhang, Z.Z.; Guo, D.A.; Li, C.L.; Zheng, J.H.; Kazuo, K.; Jia, Z.H.; Tamotsu, N. Studies on the lignan glycosides from gaultheria Yunnanensis. Yaо Xue Xue Bao 1999, 34, 128-131.

16. Kanchanapoom, T.; Kasai, R.; Yamasaki, K. Iridoid and phenolic diglycosides from Canthium berberidifolium. Phytochemistry 2002, 61, 461-464.

17. Kanchanapoom, T.; Kasai, R.; Yamasaki, K. Iridoid and phenolic glycosides from Morinda coreia. Phytochemistry 2002, 59, 551-556.

18. Gan, M.L.; Zhu, C.G.; Zhang, Y.L.; Zi, J.C.; Song, W.X.; Yang, Y.C.; Shi, J.G. Constituents from a water-soluble portion of ethanolic extract of Iodes cirrhosa. Zhongguo Zhong Yao Za Zhi 2010, $35,456-466$.

19. Inoshiri, S.; Sasaki, M.; Kohda, H.; Otsuka, H.; Yamasaki, K. Aromatic glycosides from Berchemia racemosa. Phytochemistry 1987, 26, 2811-2814. 
20. D’Abrosca, B.; DellaGreca, M.; Fiorentino, A.; Monaco, P.; Previtera, L.; Simonet, A.M.; Zarrelli, A. Potential allelochemicals from Sambucus nigra. Phytochemistry 2001, 58, 1073-1081.

21. Kitajima, J.; Tanaka, Y. Constituents of Prunus zippeliana leaves and branches. Chem. Pharm. Bull. 1993, 41, 2007-2009.

22. Arevalo, C.; Ruiz, I.; Piccinelli, A.L.; Campone, L.; Rastrelli, L. Phenolic Derivatives from the Leaves of Martinella obovata (Bignoniaceae). Nat. Prod. Comm. 2011, 6, 957-960.

Sample Availability: Not available.

(C) 2012 by the authors; licensee MDPI, Basel, Switzerland. This article is an open access article distributed under the terms and conditions of the Creative Commons Attribution license (http://creativecommons.org/licenses/by/3.0/). 